\title{
HIPERTENSÃO EM ESTUDANTES DA REDE PÚBLICA DE VITÓRIA/ES: INFLUÊNCIA DO SOBREPESO E OBESIDADE
}

\author{
HYPERTENSION IN PUBLIC SCHOOL STUDENTS FROM VITÓRIA/ES: \\ INFLUENCE OF OVERWEIGHT AND OBESITY \\ HIPERTENSIÓN EN ESTUDIANTES DE LA RED PÚBLICA DE VITÓRIAVES: \\ INFLUENCIA DEL SOBREPESO Y LA OBESIDAD
}

Artigo Original

Original ARTICLE

Artículo Original

\section{Jóctan Pimentel Cordeiro ${ }^{1}$ (Educador Físico) \\ Suelen Borges Dalmaso' (Educador Físico) \\ Sarah Amorim Anceschi ${ }^{1}$ (Graduanda em Educação Física) (Educador Físico) \\ Lucas Guimarães Ferreira \\ (Educador Físico) (Farmacêutica) \\ André Soares Leopoldo ${ }^{1}$ \\ (Educador Físico) \\ Ana Paula Lima-Leopoldo \\ (Educador Físico)}

Felipe Gonçalves dos Santos de Sá

Márcia Regina Holanda da Cunha'

1. Universidade Federal do Espírito Santo, Departamento de Desportos, Centro de Educação Física e Desportos, Vitória, ES, Brasil.

\section{Correspondência:}

Ana Paula Lima-Leopoldo.

Universidade Federal do Espírito Santo, Departamento de Desportos, Centro de Educação Física e Desportos. Av. Fernando Ferrari, 514, Goiabeiras, Vitória, ES, Brasil. 29075-910. ana.leopoldo@ufes.br

\section{RESUMO}

Introdução: Pesquisas demonstram que a obesidade pode ter início na infância. Além disso, o excesso de peso presente em $50 \%$ na infância é fator preditor da obesidade na vida adulta. As consequências são preocupantes pela associação com diversas comorbidades, entre elas, a hipertensão arterial. Estudos mostram que crianças obesas apresentam risco superior de hipertensão arterial em relação às não obesas. Objetivo: Identificar a prevalência de hipertensão arterial e sua relação com o sobrepeso e obesidade em crianças e adolescentes estudantes da rede pública do município de Vitória, ES, Brasil. Foram estudados 477 alunos, com idade entre 7 e 17 anos, sendo $42,56 \%$ e 57,44\% dos gêneros masculino e feminino, respectivamente, selecionados por meio de amostragem estratificada por conglomerados. Métodos: Na análise do perfil antropométrico foram utilizados: percentual de gordura por meio de medida de dobras cutâneas e índice de massa corporal (IMC). Além disso, foi realizada aferição da pressão arterial para classificação da hipertensão arterial. Resultados: Os resultados mostram nos estudantes de Vitória, ES, com sobrepeso, valores pressóricos elevados (limítrofes e hipertensos 1 e 2), representando nos gêneros masculino e feminino 21,1\% (PAS: $112 \pm$ 10,4 mmHg e PAD: 65,6 \pm 8,91 mmHg) e 19,2\% (PAS: $108 \pm 12,1 \mathrm{mmHg}$ e PAD: 65,6 $\pm 8,90 \mathrm{mmHg}$ ), respectivamente. No entanto, nos estudantes obesos, a prevalência foi maior, abrangendo 26,3\% (PAS: $113 \pm 11,3 \mathrm{mmHg}$ e PAD: $67,8 \pm 10,1 \mathrm{mmHg}$ ) no gêneros masculino e 25\% (PAS: $108 \pm$ 14,4 mmHg e PAD: 68,3 \pm 10,2 mmHg) no feminino. Conclusão: Os achados relatados neste estudo mostram altos índices de crianças e adolescentes acometidos tanto por excesso de peso quanto pela hipertensão arterial. Estes resultados sugerem a implementação de medidas preventivas e de tratamento dos fatores de risco cardiovascular em crianças e adolescentes do município de Vitória, ES.

Descritores: sobrepeso; obesidade; hipertensão; criança; adolescente.

\section{ABSTRACT}

Introduction: Researches show that obesity can start in childhood. Furthermore, the excess of body weight, present at $50 \%$ in childhood, is a predictor of obesity in adulthood. Consequences are alarming due to the association with several comorbidities, among them, hypertension. Studies show that obese children are at higher risk for hypertension compared to non-obese population. Objective: To identify the prevalence of hypertension and its relation to overweight and obesity in children and adolescents from public schools of the municipality of Vitória, ES, Brazil. We studied 477 students, aged between 7 and 17 years, $42.56 \%$ male and $57.44 \%$ female, who were randomly selected by stratified cluster sample. Methods: In the analysis of anthropometric profile the following were used: percentage of body fat by measuring skinfold thickness and body mass index (BMI). Moreover, measurement of blood pressure was performed to classify the arterial hypertension. Results: The results show in overweight students of Vitória, ES, high blood pressure values (pre-hypertension and stages 1 and 2 hypertension), representing 21.1\% in males (SBP: $112 \pm 10.4 \mathrm{mmHg}$ and DBP: $65.6 . \pm 8.91 \mathrm{mmHg}$ ) and 19.2\% in females (SBP: $108 \pm 12.1 \mathrm{mmHg}$ and DBP: $65.6 \pm$ $8.90 \mathrm{mmHg}$ ), respectively. However, in obese students, the prevalence was higher, comprising 26.3\% in males (SBP: $113 \pm 11.3 \mathrm{mmHg}$ and DBP: $67.8 \pm 10.1 \mathrm{mmHg}$ ) and 25\% in females (SBP: $108 \pm 14.4 \mathrm{mmHg}$ and DBP: $68.3 \pm 10.2$ $\mathrm{mmHg}$ ). Conclusion: The findings reported in this study show high rates of children and adolescents affected by both overweight and hypertension. These results suggest the implementation of preventive measures and treatment of cardiovascular risk factors in children and adolescents from the municipality of Vitória, ES.

Keywords: overweight; obesity; hypertension; child; adolescent.

\section{RESUMEN}

Introducción: La investigación ha demostrado que la obesidad puede comenzar en la infancia. Además, el peso excesivo presente en $50 \%$ de los niños en edad escolar es un factor predictivo de la obesidad en la vida adulta. Las consecuencias son preocupantes por la asociación con varias comorbilidades, entre ellas, la hipertensión arterial. Los estudios demuestran que los niños obesos tienen un mayor riesgo de hipertensión en comparación con los no obesos. Objetivo: Identificar la prevalencia de la hipertensión y su relación con el sobrepeso y la obesidad en niños y adolescentes de la red pública municipal de Vitória, ES, Brazil. Se estudiaron 477 estudiantes, con edades 
comprendidas entre los 7 y 17 años, 42,56\% y 57,44\%, hombres y mujeres, respectivamente, seleccionados mediante muestreo por conglomerado estratificado. Métodos: Fueron usados en el análisis del perfil antropométrico: porcentaje de grasa mediante la medición de pliegues cutáneos y el indice de masa corporal (IMC). Además, se realizó la medición de la presión arterial para la clasificación de la hipertensión arterial. Resultados: Los resultados muestran en los estudiantes de Vitória, ES, con sobrepeso, valores de presión sanguínea elevados (borderline e hipertensos 1 y 2), en representación de los hombres y mujeres $21,1 \%$ (PAS: $112 \pm 10,4 \mathrm{mmHg}$ y PAD: $65,6 \pm 8,91 \mathrm{mmHg}$ ) y 19,2\% (PAS: $108 \pm 12,1 \mathrm{mmHg}$ y la PAD: 65,6 $\pm 8,90 \mathrm{~mm} \mathrm{Hg}$ ), respectivamente. Sin embargo, en los estudiantes obesos, la prevalencia fue mayor, comprendiendo 26,3\% (PAS: $113 \pm 11,3 \mathrm{mmHg}$ y PAD: $67,8 \pm 10,1 \mathrm{mmHg}$ ) en el sexo masculino y 25\% (PAS: $108 \pm 14,4 \mathrm{mmHg}$ y la PAD: 68,3 $\pm 10,2 \mathrm{mmHg}$ ) en el sexo femenino. Conclusión: Los resultados reportados en este estudio muestran altos índices de niños y adolescentes afectados por tanto el sobrepeso como por la presión arterial alta. Estos resultados sugieren la aplicación de medidas preventivas y de tratamiento de los factores de riesgo cardiovascular en niños y adolescentes en el municipio de Vitória, ES.

Descriptores: sobrepeso; obesidad; hipertensión; niño; adolescente.

\section{INTRODUÇÃO}

No ano de 2011, mais de 40 milhões de crianças abaixo de cinco anos de idade estavam acima do peso. O sobrepeso e a obesidade estão em ascensão em países em desenvolvimento, especialmente em ambientes urbanos. Mais de 30 milhões de crianças com excesso de peso vivem em países em desenvolvimento e 10 milhões nos países desenvolvidos ${ }^{1}$. No Brasil esse quadro não é diferente. De acordo com a Pesquisa de Orçamentos Familiares (POF) realizada pelo Instituto Brasileiro de Geografia e Estatística (IBGE) houve progressiva redução da desnutrição infantil nas últimas décadas. Em contrapartida, o sobrepeso e a obesidade vêm aumentando². Em 2009, uma em cada três crianças de cinco a nove anos estava acima do peso recomendado pela Organização Mundial de Saúde (OMS). Entre as meninas e moças de 10 a 19 anos o crescimento do excesso de peso passou de 7,6\% (1974-75) para 19,4\% (2008-09). Nos meninos e rapazes o aumento foi ainda maior, passando de 3,7\% para $21,7 \%^{2}$.

Pesquisas demonstram que a obesidade pode ter início na infância. De acordo com Freedman et al. ${ }^{3}$, o sobrepeso presente em 50\% da infância é fator preditor da obesidade na vida adulta. Atualmente, a obesidade infantil destaca-se como grave problema de saúde pública, com início em idades cada vez mais precoces. As consequências da obesidade infantil também são preocupantes pela confirmação das morbidades associadas, como as dislipidemias, o diabetes mellitus tipo 2 e a hipertensão arterial. No Brasil, o diabetes mellitus e a hipertensão arterial constituem a principal causa de atendimentos hospitalares no sistema público de saúde ${ }^{4}$. Mundialmente, a hipertensão é o primeiro fator de risco de mortalidade, antes mesmo do tabagismo e das dislipidemias².

Dentre as morbidades associadas à obesidade, podemos destacar a hipertensão infantil, a qual está correlacionada ao crescimento da obesidade durante a infância, causada pela potencialização de hábitos alimentares inadequados e sedentarismo. Segundo alguns autores, as crianças obesas apresentam risco de duas a três vezes superior de hipertensão arterial em relação as não obesas ${ }^{3,5}$. Desta forma, à medida que a obesidade na infância aumenta, cresce também, a prevalência da hipertensão arterial infanti $\left.\right|^{5,6}$.

Estudos têm demonstrado que crianças com níveis de pressão arterial elevados apresentam maior probabilidade de se tornarem adultos portadores de hipertensão arterial ${ }^{7,8}$. Li et al. ${ }^{9}$ mostram que elevados valores iniciais de pressão arterial sistólica e diastólica durante a infância estão correlacionados positivamente com valores aumentados, quatro anos mais tarde. No entanto, a prevalência de hipertensão arterial na infância em diversos municípios é desconhecida, um dos motivos que podem maquiar a real situação pressórica das crianças e adolescentes é a falta de exames frequentes nessas faixas etárias ${ }^{10}$. O difícil diagnóstico da hipertensão arterial na infância ocorre muitas vezes pela não manifestação clínica em idades mais baixas.

Considerando que pesquisas têm mostrado o crescimento da obesidade infantil acompanhado do aumento da hipertensão arterial na infância, torna-se necessária a avaliação da pressão arterial nesta população. Embora tais informações sejam de fundamental importância para a sociedade, poucos estudos mostram relação direta entre a hipertensão arterial e a obesidade em crianças e adolescentes escolares na rede pública de Vitória, ES, Brasil. O objetivo do presente estudo foi verificar os valores pressóricos dos escolares da rede pública do município de Vitória, ES. Além disso, identificar a prevalência de hipertensão arterial e sua relação com o sobrepeso e obesidade.

\section{MÉTODOS}

Este trabalho consiste em estudo de levantamento observacional e de campo, transversal de base populacional, que visou caracterizar a prevalência de hipertensão arterial e sua relação com o sobrepeso e obesidade em uma amostra representativa de escolares do ensino público fundamental do município de Vitória, ES.

A população utilizada no presente estudo foi de alunos de ambos os gêneros, com faixa etária de 7 a 17 anos, regularmente matriculados em 16 escolas públicas do município de Vitória, ES. Para a seleção da amostra foi realizado, inicialmente, um levantamento para identificação do número de escolares matriculados na rede pública municipal de ensino no ano letivo de 2011. O cálculo amostral foi realizado com uma prevalência (P) estimada em 50\% para a ocorrência de fatores de risco cardiovascular, como hipertensão, precisão de $5 \%$ e um nível de confiança de $95 \%{ }^{11}$. Sendo assim, a amostra necessária para se avaliar neste estudo seria de 384 escolares, no entanto, utilizou-se uma amostra estratificada por conglomerados de 477 estudantes das oito regiões do município de Vitória, ES.

Os alunos participantes da pesquisa receberam o Termo de Consentimento Livre e Esclarecido (TCLE) para serem autorizados por seus responsáveis, no qual foram informados dos objetivos e procedimentos desta investigação. Os pais ou responsáveis concordaram com a participação do aluno e assinaram o TCLE, conforme normatiza a lei 196/96 que trata de pesquisas envolvendo seres humanos. Os dirigentes das escolas também foram esclarecidos previamente sobre a metodologia utilizada, com a finalidade de adequar a operacionalização das ações, sem interferir no andamento das atividades escolares. Este estudo foi aprovado pelo Comitê de Ética em Pesquisa da Universidade Federal do Espírito Santo - Centro de Ciências da Saúde, com protocolo de número 123/2011. 
O perfil antropométrico foi determinado com a finalidade de identificar a prevalência de sobrepeso e obesidade.

a. Pesagem corporal $(\mathrm{kg})$ : a pesagem foi realizada com as crianças e adolescentes descalços, vestindo uniforme escolar, em uma balança (Bioland modelo EB9015, Brasil), com capacidade de 0-150 Kg e precisão de 100g;

b. Estatura ( $m$ ): a coleta da estatura foi realizada em crianças e adolescentes colocados descalços, em posição ereta, encostados numa superfície plana vertical, braços pendentes com as mãos espalmadas sobre as coxas, os calcanhares unidos e as pontas dos pés afastadas, formando ângulo de $60^{\circ}$, joelhos em contato, cabeça ajustada ao plano de Frankfurt e em inspiração profunda. Utilizou-se estadiômetro portátil (Cardiomed ${ }^{\circledR}$, WCS modelo Wood Transportável, Curitiba/PR, Brasil), com capacidade de $20 \mathrm{~cm}$ a $200 \mathrm{~cm}$ e precisão de $0,1 \mathrm{~cm}^{12}$. O IMC foi calculado por meio da divisão do peso corporal pela estatura ao quadrado.

c. Percentual de gordura corporal: obtido por meio da técnica duplamente indireta de pregas cutâneas (DOC). Foram medidas as pregas cutâneas da região triciptal, no ponto médio entre o acrômio e o olécrano, e subescapular, a dois centímetros abaixo do bordo inferior da escápula, com o uso de um adipômetro científico de marca Cescorf (modelo Top Tec, Porto Alegre/RS, Brasil), com escalas de 0,1 e pressão constante aproximada de $10 \mathrm{~g} / \mathrm{mm}^{2}$ independente de sua abertura. As medidas de espessura foram sempre realizadas no hemicorpo direito do avaliado, utilizando o dedo indicador e o polegar da mão esquerda para diferenciar o tecido adiposo subcutâneo do tecido muscular. Todas as medidas foram realizadas com o avaliado em posição ortostática e em repouso ${ }^{13}$.

Para a mensuração do percentual de gordura corporal (\%G) utilizou-se a equação de regressão apresentada por Lohman ${ }^{14}$, para estimar a gordura corporal relativa em crianças e jovens de 7 a 17 anos ${ }^{14,15}$ : \%GC $=1,35$ $(T R+S E)-0,012(T R+S E)^{2}-C$, onde: TR = depósito de gordura triciptal; SE $=$ depósito de gordura subescapular e $\mathrm{C}=$ constantes por gênero e idade. Utilizou-se as constantes sugeridas por Lohman ${ }^{14} \mathrm{e}$ por Pires Neto e Petroski apud Arruda e Lopes ${ }^{16}$, com o intuito de facilitar e dar maior precisão na estimação do \%G. A classificação do percentual de gordura corporal foi realizada de acordo com o proposto por Lohman apud Pitanga ${ }^{17}$, conforme visualizado no Quadro 1.

A mensuração da pressão arterial (PA) foi realizada no ambiente escolar, com explicação e demonstração dos procedimentos que iriam ser realizados e de acordo com as recomendações da $V$ Diretrizes Brasileiras de Hipertensão Arterial| ${ }^{18}$. Tais procedimentos incluíram a condição inicial de repouso, com o avaliado sentado, o braço esquerdo na posição supina e apoiado na altura do coração. $O$ equipamento utilizado para aferir a pressão arterial foi um aparelho automático da marca Omrom (modelo HEM-712C, Kyoto, Japão), previamente calibrado com manguitos proporcionais à circunferência do braço da criança ou adolescente. Foram mensuradas a pressão arterial sistólica (PAS) e diastólica (PAD), sendo as medidas aferidas durante os períodos escolares: matutino (7:00 às 11:30 h) e vespertino (12:30 às 17:30 h), com intervalos de um minuto em cada verificação. $O$ agendamento foi realizado de acordo com a disponibilidade de horário que cada

Quadro 1. Classificação do índice de adiposidade.

\begin{tabular}{|c|c|c|}
\hline Classificação & Masculino & Feminino \\
\hline Muito baixo & $\leq 6,0$ & $\leq 12,0$ \\
\hline Baixo & 6,1 a 10,0 & 12,1 a 15,0 \\
\hline Ótimo & 10,1 a 20,0 & 15,1 a 25,0 \\
\hline Moderadamente alto & 20,1 a 25,0 & 25,1 a 30,0 \\
\hline Alto & 25,1 a 31,0 & 30,1 a 35,5 \\
\hline Muito alto & $>31,1$ & $>35,6$ \\
\hline
\end{tabular}

escola ofertava, podendo ser antes ou depois do intervalo e/ou até mesmo nas aulas de Educação Física. Deste modo, foram considerados 5 min de descanso sentado do avaliado em condições de repouso, e mínimo de 10 a 15 min caso estivesse realizando atividades físicas. Na aferição da PA, os valores obtidos que apresentavam diferença maior que $5 \mathrm{mmHg}$ na pressão arterial sistólica e/ou diastólica entre as duas medidas, foi realizada uma terceira aferição.

A classificação da PA foi realizada de acordo com a idade, gênero e percentil de estatura e em conformidade com a $\vee$ Diretrizes de $\mathrm{Hi}$ pertensão Arterial| ${ }^{18}$, de acordo com o The Fourth Report on The Diagnosis, Evaluation, and Treatment of High Blood Pressure in Children and Adolescents, para crianças de 1 a 17 anos, de ambos os sexos. Dessa forma, os escolares foram classificados a partir da mensuração da PA em normotensos: PAS e/ou PAD abaixo do percentil 90; limítrofes (ou pré-hipertensão): percentil entre 90 e 95; valores iguais ou superiores a 120/80 mmHg, mesmo que inferior ao percentil 90, o escolar foi considerado nesta condição. Os avaliados com o percentil igual ou superior a 95 foram classificados com hipertensão arterial. Hipertensão arterial 1: percentil entre 95 e 99 com valores pressóricos acrescidos de $5 \mathrm{mmHg}$; hipertensão arterial 2: percentil acima de 99 com valores pressóricos acrescidos de $5 \mathrm{mmHg}$.

\section{Análise Estatística}

O tamanho amostral, a tabulação, descrição e a análise dos dados foram realizados utilizando programa SigmaStat - versão 3.5 e software Microsoft Excel 2007. Para a comparação das características antropométricas entre os grupos etários e gênero foi empregada a análise de variância (ANOVA) para dois fatores, complementada com o teste post hoc de comparações múltiplas de Bonferroni. Utilizou-se o teste $t$ de Student para amostras independentes para comparar os resultados gerais médios das varáveis analisadas. O coeficiente de correlação linear de Pearson foi utilizado para avaliar o nível de correlação entre as variáveis testadas. A comparação das prevalências de normotensos, limítrofes e hipertensos entre os gêneros foi realizada de forma independente, utilizando-se o teste qui-quadrado. O nível de significância adotado foi de $5 \%(a=0,05)$.

\section{RESULTADOS}

Foram avaliados um total de 477 alunos, sendo 203 do gênero masculino e 274 do gênero feminino, entre as faixas etárias de 7 a 17 anos. A Tabela 1 ilustra as características antropométricas dos escolares avaliados na rede pública do município de Vitória, ES. Os resultados demonstram que houve interação entre idade e gênero para o peso corporal $(p<0,05)$, onde o gênero feminino apresenta maior peso corporal na faixa etária de 9 anos, no entanto, com 15 e 17 anos há redução do peso corporal em relação ao gênero masculino. Além disso, houve interação entre a estatura e o gênero $(p<0,05)$, com os meninos apresentando valores mais altos do que as meninas a partir da faixa etária de 14 anos. Enquanto, na faixa etária de 9 anos houve diferença estatística para o IMC das meninas em relação aos meninos $\left(I M C_{\text {feminino }}\right.$ $>\mid M C_{\text {masculino, }}$, na faixa etária de 10 anos o comportamento foi inverso, representado por maior IMC no gênero masculino. Considerando a média geral, não houve diferença para o peso corporal, estatura e IMC entre os gêneros.

A Tabela 2 ilustra a espessura de dobras cutâneas, subescapular e triciptal, e o percentual de adiposidade dos estudantes de acordo com gênero e idade. Em relação às espessuras das dobras cutâneas, subescapular e tricipital, foram observadas interação entre a idade e o gênero. A espessura da dobra cutânea triciptal no gênero feminino apresentou maiores valores e significativos para participantes de 9, 14 e 15 anos 
Tabela 1. Características gerais dos escolares avaliados.

\begin{tabular}{|c|c|c|c|c|c|c|}
\hline \multirow{2}{*}{ Idade (anos) } & \multicolumn{2}{|c|}{ Peso corporal (Kg) } & \multicolumn{2}{|c|}{ Estatura (m) } & \multicolumn{2}{|c|}{ IMC (Kg/m2) } \\
\hline & Masculino & Feminino & Masculino & Feminino & Masculino & Feminino \\
\hline 7 & $31,5 \pm 6,53$ & $26,7 \pm 5,61$ & $1,28 \pm 0,04$ & $1,26 \pm 0,05$ & $19,1 \pm 2,77$ & $16,7 \pm 2,96$ \\
\hline 8 & $29,8 \pm 5,20$ & $31,1 \pm 6,00$ & $1,32 \pm 0,05$ & $1,32 \pm 0,04$ & $16,9 \pm 1,77$ & $17,9 \pm 3,46$ \\
\hline 9 & $33,0 \pm 5,63$ & $39,0 \pm 11,3^{*}$ & $1,37 \pm 0,06$ & $1,39 \pm 0,07$ & $17,5 \pm 2,36$ & $20,0 \pm 4,34^{*}$ \\
\hline 10 & $41,1 \pm 9,37$ & $35,8 \pm 6,41$ & $1,42 \pm 0,05$ & $1,43 \pm 0,06$ & $20,2 \pm 3,61$ & $17,4 \pm 2,32^{*}$ \\
\hline 11 & $39,5 \pm 7,24$ & $41,4 \pm 9,60$ & $1,46 \pm 0,05$ & $1,49 \pm 0,07$ & $18,5 \pm 2,25$ & $18,6 \pm 2,99$ \\
\hline 12 & $49,1 \pm 10,8$ & $50,7 \pm 13,0$ & $1,54 \pm 0,08$ & $1,55 \pm 0,06$ & $20,6 \pm 4,13$ & $20,9 \pm 4,49$ \\
\hline 13 & $53,6 \pm 15,5$ & $49,5 \pm 10,2$ & $1,58 \pm 0,09$ & $1,56 \pm 0,07$ & $21,3 \pm 4,79$ & $20,2 \pm 3,54$ \\
\hline 14 & $58,5 \pm 14,3$ & $54,4 \pm 10,4$ & $1,68 \pm 0,08$ & $1,62 \pm 0,06^{*}$ & $20,7 \pm 4,32$ & $20,8 \pm 3,36$ \\
\hline 15 & $62,4 \pm 11,6$ & $54,8 \pm 9,98^{*}$ & $1,72 \pm 0,06$ & $1,63 \pm 0,06^{*}$ & $20,9 \pm 3,13$ & $20,7 \pm 3,41$ \\
\hline 16 & $61,6 \pm 9,62$ & $55,8 \pm 9,36$ & $1,72 \pm 0,05$ & $1,61 \pm 0,05^{*}$ & $20,7 \pm 2,50$ & $21,5 \pm 3,28$ \\
\hline 17 & $64,9 \pm 7,28$ & $53,7 \pm 5,35^{*}$ & $1,72 \pm 0,07$ & $1,64 \pm 0,07^{*}$ & $21,9 \pm 2,63$ & $19,9 \pm 1,94$ \\
\hline Total & $47,7 \pm 13,3$ & $44,8 \pm 10,4$ & $1,53 \pm 0,17$ & $1,50 \pm 0,13$ & $19,8 \pm 1,62$ & $19,5 \pm 1,61$ \\
\hline
\end{tabular}

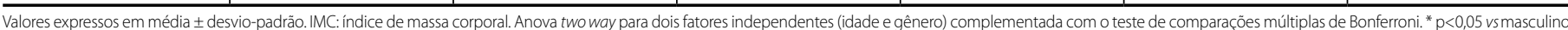

Tabela 2. Espessura de dobras cutâneas e gordura corporal relativa de escolares de acordo com gênero e idade, segundo Lohman ${ }^{14}$

\begin{tabular}{|c|c|c|c|c|c|c|}
\hline \multirow{2}{*}{ Idade (anos) } & \multicolumn{2}{|c|}{ Triciptal (mm) } & \multicolumn{2}{|c|}{ Subescapular (mm) } & \multicolumn{2}{|c|}{ Gordura corporal (\%) } \\
\hline & Masculino & Feminino & Masculino & Feminino & Masculino & Feminino \\
\hline 7 & $11,9 \pm 2,85$ & $12,5 \pm 3,46$ & $10,3 \pm 6,41$ & $10,1 \pm 5,39$ & $20,0 \pm 6,43$ & $22,2 \pm 6,01$ \\
\hline 9 & $10,7 \pm 3,82$ & $15,1 \pm 6,70^{*}$ & $9,03 \pm 5,61$ & $16,1 \pm 9,64^{*}$ & $16,9 \pm 6,40$ & $25,6 \pm 7,55^{*}$ \\
\hline 10 & $14,6 \pm 6,33$ & $11,4 \pm 2,57$ & $13,8 \pm 8,92$ & $8,96 \pm 3,81^{*}$ & $21,7 \pm 9,15$ & $19,8 \pm 4,48$ \\
\hline 11 & $11,1 \pm 3,56$ & $13,4 \pm 3,91$ & $8,58 \pm 2,88$ & $11,3 \pm 5,60$ & $16,8 \pm 5,19$ & $22,4 \pm 6,10^{*}$ \\
\hline 14 & $10,7 \pm 5,20$ & $16,0 \pm 5,87^{*}$ & $10,8 \pm 7,85$ & $13,8 \pm 5,57$ & $16,0 \pm 6,57$ & $24,8 \pm 5,52^{*}$ \\
\hline 15 & $8,94 \pm 4,19$ & $15,1 \pm 5,64^{*}$ & $10,4 \pm 4,00$ & $14,9 \pm 6,35^{*}$ & $14,8 \pm 5,63$ & $24,4 \pm 5,38^{*}$ \\
\hline 16 & $10,5 \pm 5,44$ & $15,3 \pm 5,35^{*}$ & $10,3 \pm 2,80$ & $14,5 \pm 7,44$ & $15,8 \pm 5.53$ & $23,8 \pm 4,79^{*}$ \\
\hline 17 & $9,39 \pm 5,03$ & $14,2 \pm 4,80$ & $10,1 \pm 2,91$ & $12,5 \pm 2,53$ & $14,6 \pm 6,57$ & $22,7 \pm 4,77^{*}$ \\
\hline Total & $11,5 \pm 1,91$ & $13,8 \pm 1,95^{*}$ & $10,8 \pm 2,41$ & $12,8 \pm 2,21$ & $17,3 \pm 2,38$ & $23,2 \pm 1,70^{*}$ \\
\hline
\end{tabular}

Valores expressos em média \pm desvio-padrão. Anova two way para dois fatores independentes (idade e gênero) complementada com o teste de comparaçöes múltiplas de Bonferroni. * $p<0,05$ vs masculino.

em relação ao masculino. Considerando a dobra cutânea subescapular, observa-se valores elevados no gênero feminino para participantes de 9 e 15 anos. No entanto, as meninas com 10 anos de idade apresentam menores valores de dobra subescapular em relação ao gênero masculino. Em relação ao percentual de gordura corporal também visualiza-se interação entre a idade e o gênero. $O$ gênero feminino apresentou maiores valores de gordura corporal relativa que o masculino para as todas as faixas etárias $(p<0,05)$, com exceção apenas para as idades de 7 e 10 anos. Observando desta forma, valores médios elevados de percentual de gordura no gênero feminino em relação ao masculino.

As Tabelas 3 e 4 mostram a classificação dos estudantes de acordo com os níveis de adiposidade, considerando o gênero e faixa etária conforme sugerido por Lohman ${ }^{14}$. De acordo com análise conjunta de gêneros, os resultados demonstram elevada prevalência de alunos classificados como moderadamente alto $(n=71 ; 14,8 \%)$ e alto $(n=86$; 18\%), representando sobrepeso e obesidade, respectivamente.

A Tabela 5 ilustra os valores pressóricos (PAS e PAD) dos estudantes de acordo com gênero e classificação de adiposidade. Em relação aos valores de PA não foram observadas interação entre adiposidade e gênero. Na condição eutrófico, houve menor valor de PAS no gênero feminino em relação ao masculino $(p<0,05)$. Considerando ambos os gêneros (intragrupo), visualiza-se diferença significativa na PAD entre os eutróficos e obesos, as quais foram elevadas no grupo obeso.

Na avaliação de possíveis relações entre variáveis dependentes (PAS e PAD) e independentes (IMC e \%G), observou-se correlação fraca, no entanto, significativa para a PAD $(p<0,05)$. Para PAS não houve correlação com IMC e \%G (Tabela 6).
Tabela 3. Classificação de adiposidade de estudantes do gênero feminino.

\begin{tabular}{c|c|c|c|c|c|c|c|c}
\hline \multirow{2}{*}{$\begin{array}{c}\text { Idade } \\
\text { (anos) }\end{array}$} & \multicolumn{7}{|c|}{ Classificação de Adiposidade } \\
\cline { 2 - 9 } & \multicolumn{2}{|c|}{ Baixo } & \multicolumn{2}{c|}{ Ótimo } & \multicolumn{2}{c}{ Mod. alto } & \multicolumn{2}{c}{ Alto } \\
\cline { 2 - 9 } & $\mathbf{n}$ & $\%$ & $\mathbf{n}$ & $\%$ & $\mathbf{n}$ & $\%$ & $\mathbf{n}$ & $\%$ \\
\hline 7 & 3 & 23,0 & 8 & 61,5 & 1 & 7,7 & 1 & 7,8 \\
\hline 8 & 5 & 23,8 & 11 & 52,3 & 2 & 9,5 & 3 & 14,4 \\
\hline 9 & 2 & 6,6 & 13 & 43,3 & 4 & 13,5 & 11 & 36,6 \\
\hline 10 & 3 & 13,6 & 16 & 72,8 & 3 & 13,6 & 0 & - \\
\hline 11 & 3 & 13,6 & 11 & 50,0 & 5 & 22,8 & 3 & 13,6 \\
\hline 12 & 1 & 4,0 & 12 & 46,0 & 8 & 30,7 & 5 & 19,3 \\
\hline 13 & 4 & 11,0 & 18 & 50,0 & 7 & 19,5 & 7 & 19,5 \\
\hline 14 & 0 & - & 20 & 60,5 & 4 & 12,2 & 9 & 27,3 \\
\hline 15 & 0 & - & 22 & 56,4 & 11 & 28,2 & 6 & 15,4 \\
\hline 16 & 0 & - & 15 & 68,2 & 4 & 18,2 & 3 & 13,6 \\
\hline 17 & 0 & - & 7 & 70,0 & 3 & 30,0 & 0 & - \\
\hline Total & 21 & 7,7 & 153 & 55,8 & 52 & 19,0 & 48 & 17,5 \\
\hline
\end{tabular}
n: número de alunos. Mod. alto: moderadamente alto.

A pressão arterial dos alunos considerados obesos foi classificada levando em consideração os níveis de adiposidade (Figura 1A). Observa-se no gênero masculino prevalência de $21 \%$ de hipertensão, classificados em hipertensão 1 (18,4\%) e hipertensão 2 (2,6\%). No gênero feminino $14,6 \%$ foram classificados com hipertensão, compreendidos em hipertensão 1 (12,5\%) e hipertensão 2 (2,1\%). Os limítrofes representam 5,3\% e 10,4\% nos gêneros masculino e feminino, respectivamente.

A Figura 1B ilustra a categorização pressórica de alunos classificados com sobrepeso. Observa-se no gênero masculino prevalência de $21,1 \%$ de hipertensão, compreendidos em hipertensão 1 (15,8\%) 
Tabela 4. Classificação de Adiposidade de estudantes do gênero masculino.

\begin{tabular}{c|c|c|c|c|c|c|c|c}
\hline & \multicolumn{7}{c|}{ Classificação de Adiposidade } \\
\hline $\begin{array}{c}\text { Idade } \\
\text { (anos) }\end{array}$ & \multicolumn{2}{|c|}{ Baixo } & \multicolumn{2}{c|}{ Ótimo } & \multicolumn{2}{c}{ Mod. alto } & \multicolumn{2}{c}{ Alto } \\
\hline & $\mathbf{n}$ & $\%$ & $\mathbf{n}$ & $\%$ & $\mathbf{n}$ & $\%$ & $\mathbf{n}$ & $\%$ \\
\hline 7 & 0 & - & 4 & 66,6 & 0 & - & 2 & 33,4 \\
\hline 8 & 0 & - & 16 & 94,1 & 0 & - & 1 & 5,9 \\
\hline 9 & 4 & 13,8 & 16 & 55,0 & 5 & 17,2 & 4 & 14,0 \\
\hline 10 & 3 & 14,2 & 7 & 33,3 & 2 & 9,5 & 9 & 43,0 \\
\hline 11 & 1 & 5,0 & 13 & 65,0 & 5 & 25,0 & 1 & 5,0 \\
\hline 12 & 0 & - & 14 & 56,0 & 4 & 16,0 & 7 & 28,0 \\
\hline 13 & 1 & 5,2 & 12 & 63,2 & 1 & 5,3 & 5 & 26,3 \\
\hline 14 & 6 & 22,2 & 16 & 59,3 & 0 & - & 5 & 18,5 \\
\hline 15 & 2 & 9,5 & 16 & 76,3 & 1 & 4,7 & 2 & 9,5 \\
\hline 16 & 0 & - & 8 & 89,0 & 0 & - & 1 & 11,0 \\
\hline 17 & 3 & 33,3 & 4 & 44,5 & 1 & 11,1 & 1 & 11,1 \\
\hline Total & 20 & 9,8 & 126 & 62,0 & 19 & 9,3 & 38 & 18,9 \\
\hline
\end{tabular}

n: número de alunos. Mod. alto: moderadamente alto.

Tabela 5. Valores pressóricos dos estudantes avaliados de acordo com a classificação de adiposidade.

\begin{tabular}{c|c|c|c|c}
\hline \multirow{2}{*}{ Classificação } & \multicolumn{2}{|c|}{ PAS (mmH) } & \multicolumn{2}{c}{ PAD (mmHg) } \\
\cline { 2 - 5 } & Masculino & Feminino & Masculino & Feminino \\
\hline Eutrófico & $112 \pm 15,9$ & $107 \pm 13,8^{*}$ & $61,9 \pm 10,9$ & $62,7 \pm 10,4$ \\
\hline Sobrepeso & $112 \pm 10,4$ & $108 \pm 12,1$ & $65,6 \pm 8,91$ & $65,6 \pm 8,90$ \\
\hline Obeso & $113 \pm 11,3$ & $108 \pm 14,4$ & $67,8 \pm 10,1^{\#}$ & $68,3 \pm 10,2^{\#}$ \\
\hline
\end{tabular}

Valores expressos em média \pm desvio padrão. PAS: pressão arterial sistólica; PAD: pressão arterial diastólica. Anova two-way para dois fatores independentes complementada com teste de comparaçōes múltiplas de Bonferroni. ${ }^{*} \mathrm{p}<0,05$ vs masculino. ${ }^{*} \mathrm{p}<0,05$ vs eutrófico (intragrupo).

Tabela 6. Correlação da PAS e PAD com IMC e \%G dos escolares.

\begin{tabular}{c|c|c|c|c}
\hline & \multicolumn{2}{|c|}{ IMC } & \multicolumn{2}{c}{$\% \mathrm{G}$} \\
\hline & Masculino & Feminino & Masculino & Feminino \\
\hline PAS & & & & \\
\hline$r$ & 0,032 & 0,032 & $-0,063$ & 0,072 \\
\hline$p$ & 0,65 & 0,59 & 0,37 & 0,23 \\
\hline$P A D$ & & & & \\
\hline$r$ & $0,312^{*}$ & $0,305^{*}$ & 0,178 & 0,25 \\
\hline$p$ & $<0,001$ & $<0,001$ & $<0,05$ & $<0,001$ \\
\hline
\end{tabular}

, correlação linear de Pearson; $p$, nível de significância $(p<0,05)$; PAS, pressão arterial sistólica; PAD, pressão arterial diastólica; IMC, índice de massa corporal; \%G, percentual de gordura corporal. *Correlação fraca (de r $=0,30$ a $r=0,49$.

e hipertensão 2 (5,3\%). No gênero feminino 13,4\% foram classificados com hipertensão, incluídos em hipertensão 1 (11,5\%) e hipertensão 2 (1,9\%). Os limítrofes representam 5,8\% no gênero feminino, entretanto, no gênero masculino não foram identificados alunos nesta condição.

A classificação de pressão arterial, de ambos os gêneros, em alunos considerados eutróficos está representada na figura 1C. Observa-se que 81,5\% dos alunos do gênero masculino foram considerados normotensos. No entanto, mesmo na ausência de sobrepeso e obesidade 12,3\% foram identificados como hipertensos, representando 8,2\% e 4,1\% com hipertensão 1 e 2, simultaneamente. Além disso, foram considerados como limítrofes $6,2 \%$. Comportamento semelhante foi visualizado no gênero feminino, apresentando prevalência de 78,7\%, 5,7\%, 10,3\% e 5,2\% de meninas classificadas como normotenso, limítrofe, hipertensão 1 e 2 , respectivamente.

Considerando as classificações de adiposidade em eutrófico, sobrepeso e obeso, observou-se que não houve diferença estatística na frequência de pressão arterial em ambos os gêneros (masculino, $p=0,47$; feminino, $p=0,82$ ) (Figura 1). Além disso, não houve diferença significativa entre a frequência de escolares classificados como eutróficos, sobrepeso e obeso sobre a pressão arterial, independente do gênero $(p=0,74)$
A Obeso

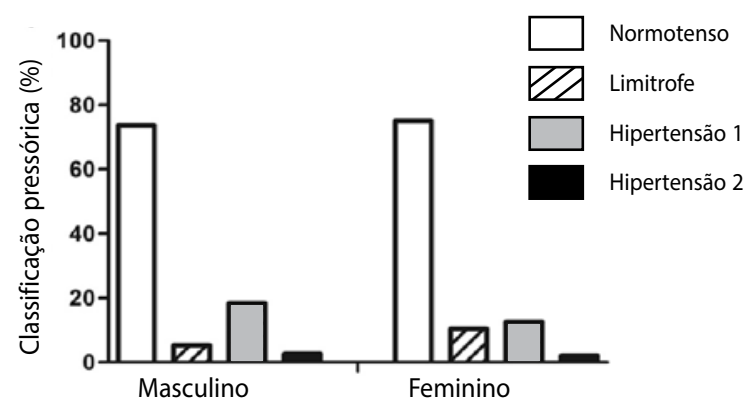

B

Sobrepeso

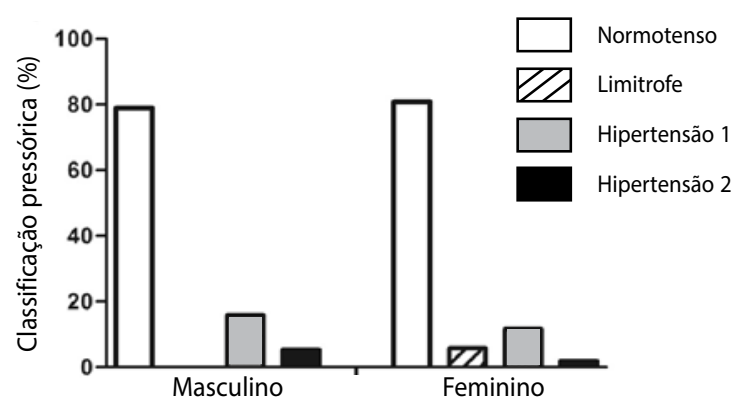

C Eutrófico

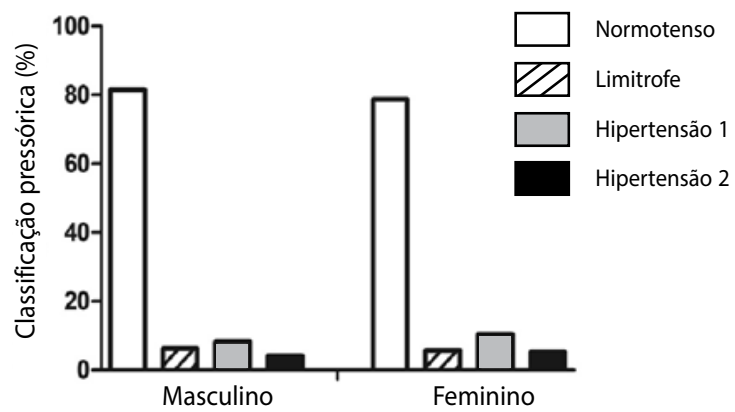

Figura 1. Classificação pressórica de estudantes dos gêneros masculino e feminino da rede pública de Vitória,ES. (A) obeso; (B) sobrepeso; (C) eutrófico. $p>0,05$. Teste do Qui-quadrado.

\section{DISCUSSÃO}

Atualmente o sobrepeso e obesidade estão em crescente desenvolvimento tanto em adultos quanto em crianças e adolescentes. Este quadro pode ser observado em escala global, caracterizando esta doença, atualmente, como pandemia ${ }^{19}$.

No Brasil, estudos realizados nas cidades Jundiaí, SP20, Londrina, $P^{21}$ e Recife, PE $^{22}$, diagnosticaram valores de $24 \%, 18,5 \%$ e $24,7 \%$ de excesso de peso, respectivamente, em crianças e adolescentes. Os achados do presente estudo são de similar expressão, apresentando elevados valores de 32,9\% de excesso de peso em ambos os gêneros, mostrando que a alta prevalência desses distúrbios metabólicos não se restringe a regiões isoladas, mas está presente em diversas regiões do território nacional.

A Pesquisa de Orçamentos Familiares (POF) realizada pelo Instituto Brasileiro de Geografia e Estatística (IBGE)² entre os anos de 2008 e 2009, aponta que o excesso de peso atinge 33,5\% das crianças de 5 a 9 anos. O estudo ressalta ainda que dentre o total de crianças avaliadas 16,6\% 
dos meninos e 11,8\% das meninas foram consideradas com obesidade. O excesso de peso representou nos adolescentes de 10 a 19 anos $21,7 \%$ e $19 \%$ no gênero masculino e feminino, respectivamente. Entre estes últimos, a obesidade compreende 5,9\% nos meninos e rapazes e $4 \%$ nas meninas e moças. A pesquisa destaca que na região Sudeste 40,3\% dos meninos e 38\% das meninas de cinco a nove anos de idade estão com excesso de peso, na mesma região a obesidade oscila entre $20-27 \%$ dos adolescentes.

Em estudo anterior desenvolvido em Vitória, ES, Dos Anjos e Muller $^{23}$, não identificaram prevalência de sobrepeso e obesidade na população estudada, apresentando distinção aos achados da POF na região Sudeste. Entretanto, outro estudo ${ }^{10}$ realizado no mesmo estado, anos mais tarde, mostra que $23,2 \%$ das crianças apresentavam excesso de peso, abrangendo 23,5\% e 22,9\% do total de meninas e meninos, respectivamente. Vale ressaltar que o método usado para a classificação foi a avaliação do IMC de acordo com Cole et al. ${ }^{24}$, distribuindo os avaliados em apenas dois grupos - com presença ou ausência de excesso de peso.

O presente estudo mostra que nos estudantes avaliados do gênero masculino do município de Vitória,ES, o sobrepeso e a obesidade representam 9,3\% e 18,9\%, respectivamente. Enquanto no gênero feminino o sobrepeso e a obesidade atingem 19\% e 17,5\%. Considerando a análise conjunta de sobrepeso e obesidade evidencia-se $28,2 \%$ e $36,5 \%$ de excesso de peso nos avaliados dos gêneros masculino e feminino, respectivamente. Pode-se observar disparidade nos valores encontrados nos estudantes, evidenciando maiores valores de excesso de peso no gênero feminino, em comparação aos achados de Molina et al. ${ }^{10}$. Um ponto a ser considerado que pode ter levado a essa discrepância, é a classificação do sobrepeso e obesidade, do atual estudo, por meio das dobras cutâneas para a medida e cálculo do percentual de gordura, o que diminui o risco de classificação equivocada, mostrando que IMC pode subestimar a classificação de excesso de peso ${ }^{25}$.

Rosa e Ribeiro ${ }^{25}$ destacam que a elevação da pressão arterial na infância e na adolescência está relacionada a incrementos fisiológicos no tamanho corporal. O peso não é fator principal para se estudar adiposidade isoladamente, uma vez que sofre variações em função de outros componentes. Em seu lugar, tem-se empregado a medida da espessura de dobras cutâneas, tornando mais fidedigna a avaliação por adiposidade 25 .

Estudos observacionais correlacionam os índices peso-estatura com a pressão arterial ${ }^{26,27}$. Os autores sugerem que adiposidade seja um fator determinante desses níveis por meio do aumento percentual de obesidade e de maior tendência à distribuição de adiposidade central. Desta forma, a hipertensão arterial é uma morbidade que apresenta estreita ligação com o sobrepeso e obesidade ${ }^{26,27}$. No entanto, os trabalhos atuais que analisam estes últimos e suas relações com a hipertensão arterial em crianças e adolescentes ainda são escassos no Brasil. Além de parte dos estudos realizados nessa perspectiva se valerem da classificação de sobrepeso e obesidade por meio do IMC, o qual não caracteriza a real situação de prevalência de adiposidade, tornando menos fiel a correlação da morbidade com a hipertensão arterial.
Considerando a correlação entre as variáveis de pressão arterial, tanto sistólica quanto diastólica, com as variáveis antropométricas avaliadas, observa-se neste estudo correlação fraca entre as seguintes variáveis: PAD e IMC ( $p<0,001)$ e PAD e \%G $(p<0,05)$. Esses achados corroboram Reuter et al. ${ }^{28}$ que mostram correlação fraca e significativa entre IMC e PAD ( $r=0,358 ; p<0,001)$ e \%G e PAD $(r=0,238 ; p<0,001)$.

Os indivíduos que apresentam alterações de pressão arterial podem ser classificados como limítrofe, hipertensão 1 e hipertensão 2, formados por grupos com valores pressóricos acima dos considerados normais para a média da população ${ }^{18}$. Molina et al. ${ }^{10}$, em dados supracitados, relata que $23,2 \%$ dos estudantes apresentam excesso de peso, dentre os quais, 6,4\% dos avaliados apresentaram, também, alteração na pressão arterial. Outro estudo ${ }^{29}$, publicado no mesmo ano, avaliou 129 crianças e adolescentes obesos com idade de 7 a 14 anos de ambos os gêneros, no período de agosto de 2005 a julho de 2006. Os indivíduos avaliados eram pacientes do programa de tratamento de obesidade infanto-juvenil oferecido pelo Sistema Único de Saúde (SUS). Os autores relatam presença de hipertensão arterial em 21,1\% da população estudada.

Corroborando com os achados dos demais estudos acima citados, observa-se nos estudantes de Vitória,ES, com sobrepeso, altos valores pressóricos representando nos gêneros masculino e feminino $21,1 \%$ e $19,2 \%$, respectivamente. Nos alunos classificados como obesos os valores mostram-se mais elevados, abrangendo 26,3\% e $25 \%$ nos gêneros masculino e feminino, respectivamente. Cabe salientar, que os valores de gordura corporal relativa entre $25 \%$ e $30 \%$, em crianças e adolescentes, estão associados ao alto risco de desenvolvimento de doenças cardiovasculares, hipertensão arterial, hipercolesterolemia e hiperlipidemia ${ }^{21}$.

Achado inusitado neste trabalho foi a alta prevalência de elevação da pressão arterial em estudantes considerados eutróficos, representando 18,5\% dos avaliados nesta condição. Tal fator pode indicar desenvolvimento de hipertensão arterial causado por inadequada alimentação e o sedentarismo. Sabe-se que o nível de pressão arterial é determinado por fatores genéticos e ambientais e que a alimentação é um dos mais importantes ${ }^{25}$. Neumann et al. ${ }^{30}$ ressaltam que componentes da dieta podem provocar efeitos adversos ao organismo e que a alimentação e a atividade física têm estreita relação com as doenças crônicas não transmissíveis, de modo que alimentos ou preparações que contêm colesterol, ácidos graxos saturados, ácidos graxos trans e sódio aumentam o risco de doenças cardiovasculares, quando consumidos em periodicidade e quantidades inadequadas.

\section{CONCLUSÂO}

Os achados relatados neste estudo mostram altos índices de crianças e adolescentes acometidos tanto por excesso de peso quanto pela hipertensão arterial. Estes resultados sugerem a implementação de medidas preventivas e de tratamento dos fatores de risco cardiovascular, em crianças e adolescentes, no município de Vitória, ES.

Todos os autores declararam não haver qualquer potencial conflito de interesses referente a este artigo.

CONTRIBUIÇÕES DOS AUTORES: Cada autor contribuiu individual e significativamente para o desenvolvimento do manuscrito. JPC (0000-0003-3814-5021)*, SBD (0000-0002-8182-7135)*, ASL (0000-0002-3131-2467)* E APLL (0000-0002-4049-4418)* foram os principais contribuintes na redação do manuscrito. JPC, SBD, SAA (00000003-4368-3027)* e FGSS (0000-0002-7285-1184)* realizaram a avaliação dos perfis antropométricos e pressóricos de todos os escolares do estudo. JPC, SAA, ASL e APLL realizaram a análise estatística dos dados. JCP, LGF (0000-0002-2970-7355), MRHC (0000-0002-7087-9482)*, ASL e APLL participaram ativamente da discussão dos resultados e versão final do trabalho. *Número ORCID (Open Researcher and Contributor ID). 


\section{REFERÊNCIAS}

1. World Health Organization (WHO). The World Health Report 2002: reducing risks, promoting health life. [Acesso em 11 ago/2013]. Disponível em: http://www.who.int/mediacentre/factsheets/fs311/ en/\#.UfaxKo9brPk.

2. Instituto Brasileiro de Geografia e Estatística (IBGE). Pesquisa de orçamentos familiares 20082009: antropometria e estado nutricional de crianças, adolescentes e adultos no Brasil. [Acesso em 15 jul/2013]. Disponível em: http://saladeimprensa.ibge.gov.br/noticias?view=noticia\&id=1\&idnoticia=1699\&busca=1\&t=pof-20082009-desnutricao-cai-peso-criancas-brasileiras-ultrapa ssa-padrao-internacional.

3. Freedman DS, Khan LK, Dietz WH, Srinivasan SR, Berenson GS. Relationship of childhood obesity to coronary heart disease risk factors in adulthood: the Bogalusa Heart Study. Pediatrics. 2001;108(3):712-8.

4. Brasil. Ministério da Saúde. Plano Nacional e Saúde - PNS 2012-2015: Textos Básicos de Saúde. [Acesso em: 10/jul/2013]. Disponível em: http://conselho.saude.gov.br/biblioteca/Relatorios/plano_nacional_saude_2012_2015.pdf.

5. Sorof J, Daniels S. Obesity hypertension in children: a problem of epidemic proportions. Hypertension. 2002;40(4):441-7.

6. Cattaneo A, Monasta L, Stamatakis E, Lioret S, Castetbon K, Frenken F, et al. Overweight and obesity in infants and pre-school children in the European Union: a review of existing data. Obes Rev. 2010;11(5):389-98.

7. Oliveira RG, Lamounier JA, Oliveira AD, Castro MD, Oliveira JS. Blood pressure in school children and adolescents - The Belo Horizonte study. J Pediatr (Rio Janeiro). 1999;75(4):256-66.

8. Fuentes RM, Notkola IL, Shemeikka S, Tuomilehto J, Nissinen A. Tracking of systolic blood pressure during childhood: a 15-year follow-up population-based family study in eastern Finland. J Hypertens. 2002;20(2):195-202.

9. Li L, Wang Y, Cao W, Xu F, Cao J. Longitudinal studies of blood pressure in children. Asia Pac J Public Health. 1995;8(2):130-3.

10. Molina Mdel C, Faria CP, Montero MP, Cade NV, Mill JG. Cardiovascular risk factors in 7-to-10-year-old children in Vitória, Espírito Santo State, Brazil. Cad Saude Publica. 2010;26(5):909-17

11. Medronho RA, Carvalho DM, Bloch KV, Luiz RR, Werneck GL. Epidemiologia. São Paulo: Atheneu; 2006.

12. Giugliano R, Melo AL. [Diagnosis of overweight and obesity in schoolchildren: utilization of the body mass index international standard]. J Pediatr (Rio Janeiro). 2004;80(2):129-34.

13. Miranda JMQ, Palmeira MV, Polito LFT, Brandão MRF, Bocalini DS, Ferreira Júnior AJ, et al. Prevalência de sobrepeso e obesidade infantil em instituições de ensino:públicas vs. privadas. Rev Bras Med Esporte 2015;21( 2):104-7.

14. Lohman TG. Applicability of body composition techniques and constants for children and youths. Exerc Sport Sci Rev. 1986;14:325-57.
15. Almeida CAN, Ciampo LAD, Ricco RG. Indicadores clínicos, antropométricos, bioquímicos e nutricionais da obesidade na adolescência [anais]. In: Simpósio de Obesidade e Anemia Carencial na Adolescência, Salvador, Bahia. Salvador: Instituto Danone; 2000. p. 77-88.

16. Arruda ELM, Lopes AS. Gordura corporal, nível de atividade física e hábitos alimentares de adolescentes da região serrana de Santa Catarina, Brasil. Rev Bras Cineantropom Desempenho Hum. 2007;9(1):05-11.

17. Pitanga FJ. Testes, medidas e avaliação em educação física e esportes. São Paulo: Phorte; 2005.

18. Sociedade Brasileira de Cardiologia, Sociedade Brasileira de Hipertensão,Sociedade Brasileira de Nefrologia. V Diretrizes Brasileiras de Hipertensão Arterial; 2006. Disponível em: http://departamentos. cardiol.br/dha/vdiretriz/vdiretriz.asp. Acesso em 04 jul/2013.

19. Ribeiro Junior HDC. A pandemia de obesidade entre os jovens. Rev Paul Pediatr 2007;25(4):304.

20. Vanzelli AS, Castro CDT, Pinto MDS, Passos SD. Prevalência de sobrepeso e obesidade em escolares da rede pública do município de Jundiaí, São Paulo. Rev Paul Pedriatr 2008;26(1):48-53.

21. Ronque ERV, Cyrino ES, Dórea VR, Júnior HS, Galdi EHG, Arruda M. Prevalência de sobrepeso e obesidade em escolares de alto nível socioeconômico em Londrina, Paraná, Brasil. Rev Nutr. 2005;6(18):709-17.

22. Balaban G, Silva GAP, Motta MEFA. Prevalência de sobrepeso e obesidade em escolares de diferentes classes socioeconômicas em Recife, PE. J Pediatr 2001;23(4):285-9.

23. Dos Anjos L, Muller EC. Prevalência da desnutrição e obesidade em escolares do ensino fundamental do município de Vitória-ES dada à condição socioeconômica. [Acesso em 14 jul/2013]. Rev Dig EFDeportes, Buenos Aires. 2006;95(11). Disponível em http://www.efdeportes.com/efd95/desnutri.htm.

24. Cole TJ, Bellizzi MC, Flegal KM, Dietz WH. Establishing a Standard Definition for Child Overweight and Obesity Worldwide: International survey. BMJ 2000;320(7244):1240-3.

25. Rosa AA, Ribeiro JP. Hipertensão arterial na infância e na adolescência: fatores determinantes. J Pediatr 1999;75(2):75-82.

26. Roberti MIDA. Níveis pressóricos arteriais de pré-escolares do município de São Paulo - influência da desnutrição proteico-energética [dissertação]. São Paulo: Universidade Federal de São Paulo, Programa de Pós-Graduação em Medicina; 1989.

27. Voors AW, Foster TA, Frerichs RR, Webber LS, Berenson GS. Studies of blood pressures in children, ages 5-14 years, in a total biracial community: the Bogalusa Heart Study. Circulation. 1976;54(2):319-27.

28. Reuter EM, Reuter CP, Burgos LT, Reckziegel MB, Nedel FB, de Albuquerque IM, et al. Obesidade e hipertensão arterial em escolares de Santa Cruz do Sul - RS, Brasil. Rev Assoc Med Bras. 2012;58(6):666-72.

29. Ferreira JS, Aydos RD. Prevalence of hypertension among obese children and adolescents. Cien Saude Colet. 2010;15(1):97-104.

30. Neumann Al, Martins IS, Marcopito LF, Araujo EA. Dietary patterns associated with risk factors for cardiovascular disease in a Brazilian city. Rev Panam Salud Publica. 2007;22(5):329-39. 